\title{
ROBUST DELAY-DEPENDENT STABILITY FOR TAKAGI-SUGENO FUZZY SYSTEMS WITH MIXED TIME-VARYING DELAYS
}

\author{
M. Parimala \\ Department of Mathematics, Bannari Amman Institute of Technology, \\ Sathyamangalam, Erode, Tamil Nadu, India. \\ R. Preetha \\ Department of Mathematics, Bannari Amman Institute of Technology, \\ Sathyamangalam, Erode, Tamil Nadu, India.
}

\section{A. Manivannan}

Division of Mathematics, Vellore Institute of Technology - Chennai Campus, Chennai, Tamil Nadu, India.

\begin{abstract}
This study presents the problem of robust stability analysis for the Takagi-Sugeno (T-S) fuzzy systems with mixed time-varying delays. In the proposed T-S fuzzy system, there occurred discrete and distributed time delays that are taken in account as mixed time varying delays. Based on the refined Lyapunov-Krasovskii functional which contains the information of the upper and lower bounds of time-varying delays, robust stability conditions of delay-dependent T-S fuzzy system are derived in terms of the linear matrix inequalities. The obtained delay-dependent conditions ensure the robustly asymptotically stability of the proposed delayed T-S fuzzy systems. Finally, numerical examples are given to show the superiority of the derived sufficient conditions.
\end{abstract}

Keywords: Delay dependent Stability, Time varying delay, Distributed delay, Lyapunov-Krasovskii functional, T-S fuzzy model, Linear matrix inequality

Cite this Article: M. Parimala, R. Preetha, A. Manivannan, Robust delay-dependent stability for Takagi-Sugeno fuzzy systems with mixed time-varying delays, International Journal of Electrical Engineering and Technology (IJEET), 12(5), 2021, pp. 18-27.

https://iaeme.com/Home/issue/IJEET?Volume=12\&Issue $=5$

\section{INTRODUCTION}

The dynamical analysis of the nonlinear systems has received more attention in the research community due to its various studies for the steady states of the systems such as stability, bifurcation, periodic and chaotic. These analyses are helpful to understanding the dynamical 
nature of the proposed dynamical models. As we know that, the dynamical analysis of nonlinear systems is difficult for analyzing the stability nature. In this situation, the T-S fuzzy approach can be applied to express the nonlinear systems as a convex combination of the local linear systems blended by IF-THEN membership functions. Therefore, a significant results have been reported in the existing research results (see [1], [2], [3], [4] ). As an example, the authors in $[5,6,7,8]$, have investigated the stability and stabilization issue for T-S fuzzy model of the proposed systems based on suitable Lyapunov-functional and expressed in terms of matrix inequalities.

On the other hand, time-delay is an unavoidable factor in the dynamical systems. It has wide applications in the field of chemical reactor systems, aircraft systems, vehicle suspension systems, neural networks, and economic-modelled control systems. This delay factor plays an import role in the dynamical nature i.e., this factor can be lead some poor performance or instability of the whole nature. Therefore, the investigation of the time-delay in the dynamical systems has received importance from the researchers in the literature $[9,10,11]$. In the general, two kinds of sufficient conditions have been derived by the researchers such as delay-dependent condition and delay-independent condition. The delay-dependent condition is considered less conservative than the independent condition when the size of delay is small, therefore, researchers have paid attention on delay-dependent stability analysis for the dynamical systems $[12,13,14,15]$.

Moreover, when modelling a dynamical system, we cannot avoided some modelling error in the designing of the systems parameters, which is called as parameter uncertainty. This uncertainty will make some poor performances in the stability of the systems. Hence, the robust stability analysis became an important research topic among the researchers $[16,17,18]$. As an example, in [16], the problem of robust stability analysis has been investigated for fractional order neutral-type nonlinear systems with actuator saturation. Moreover, the authors in [17] the cases of matrix parameters and delays uncertainties have taken in the stability analysis of linear systems with distributed delays and the robust stability conditions have been obtained based on suitable Lyapunov-functional.

To the best of the authors' knowledge, very few results are available on the delay-dependent stability analysis for T-S fuzzy systems with mixed time-varying delays and parameter uncertainties. This is the motivation of this study. According to the above discussion, in this work, delay-dependent robust stability analyses are addressed. The mixed time-varying delay and parameter uncertainties are taken in the proposed T-S fuzzy systems. Based on this, a suitable LKF is constructed which includes the information of the time-delays and their upper bounds. Then, the delay-dependent robust stability conditions are derived in the form of LMIs. Finally, the derived conditions are validated with suitable numerical examples. From the simulation results, we can confirm that the effectiveness and superiority of the derived results.

Notation: Throughout this manuscript, the n-dimensional Euclidean space is denoted by $\mathfrak{R}^{n}$ and the set of all $n \times n$ real matrices are denoted by $\mathfrak{R}^{n x n}$. The notation $Z \geq W$ (respectively, $Z>W$ ) means that, positive semi-definite (respectively, positive definite) where $Z$ and $W$ are symmetric matrices. The notation * always denotes the symmetric block in one symmetric matrix.

\section{METHODS}

Consider the t-s fuzzy model of nonlinear systems with mixed time-varying delays as below:

Plant rule $i$ : If $\gamma_{1}(t)$ is $\mu_{1}{ }^{i}$ and $\ldots$ and $\gamma_{l}(t)$ is $\mu_{l}^{i}$ Then 


$$
\begin{aligned}
& \dot{x}(t)=\left(A_{1 i}+\Delta A_{1 i}(t)\right) x(t)+\left(A_{2 i}+\Delta A_{2 i}(t)\right) x(t-\tau(t))+\left(A_{3 i}+\Delta A_{3 i}(t)\right) \int_{t-\tau(t)}^{t} x(\alpha) d \alpha \\
& x(t)=\phi(t), \quad \forall t \in\left[-\max \left\{\tau_{2}, r\right\}, 0\right], \quad i=1,2, \ldots . . r .
\end{aligned}
$$

Where $x(t)=\left[x_{1}(t), x_{2}(t), \ldots, x_{n}(t)\right] \in \quad \mathfrak{R}^{n}$ denotes the state vector. $\boldsymbol{\phi}(\cdot) \in$ $\mathbb{C}\left(\left[-\boldsymbol{m a x}\left\{\boldsymbol{\tau}_{2}, \boldsymbol{r}\right\}, \mathbf{0}\right], \mathfrak{R}^{n}\right)$ denotes the initial function. $A_{1 i}, A_{2 i}, A_{3 i} \in \mathfrak{R}^{n \times n}$ are known matrices. ${ }^{\mu_{i}^{j}}$ is the fuzzy set.r is the number of IF-THEN rules, $\gamma(t)=\left[\gamma_{1}(t), \gamma_{2}(t), \ldots, \gamma_{l}(t)\right]$ are the premise variables. $\tau(t)$ denotes the time-varying delay satisfies $0<\tau_{1} \leq \tau(t) \leq \tau_{2}, \dot{\tau}(t) \leq \mu<1$, and the distributed delay $r(t)$ satisfies the following condition $0 \leq r(t) \leq r$ where $\mu^{\tau_{1}, \tau_{2}}$ and $r$ are constants. The parameter uncertainties $\Delta A_{1 i}(t), \Delta A_{2 i}(t)$ and $\Delta A_{3 i}(t)$ are of the form

$$
\left[\Delta A_{1 i}(t) \quad \Delta A_{2 i}(t) \quad \Delta A_{3 i}(t)\right]=B_{i} C_{i}(t)\left[\begin{array}{lll}
D_{1 i} & D_{2 i} & D_{3 i}
\end{array}\right]
$$

where $B_{i}, D_{1 i}, D_{2 i}, D_{3 i}$ are known real constants matrices $C_{i}(t)$ known real constant matrices with Lebesgure measurable elements which satisfies $C_{i} T_{(t)} C_{i}(t) \leq I, \forall t \in \mathfrak{R}$. Where $I$ is the appropriate dimension identity matrix.

Then, the overall t-s fuzzy model with time varying delay (1) can be deduced as follows

$$
\dot{x}(t)=\sum_{i=1}^{r} \mu_{i}(\gamma(t))\left\{\left(A_{1 i}+\Delta A_{1 i}(t)\right) x(t)+\left(A_{2 i}+\Delta A_{2 i}(t)\right) x(t-\tau(t))+\left(A_{3 i}+\Delta A_{3 i}(t)\right) \int_{t-r(t)}^{t} x(\alpha) d \alpha\right\}
$$

$$
\mu_{i}(\gamma(t))=\frac{\eta_{i}(\gamma(t))}{\sum_{i=1}^{r} \eta_{i}(\gamma(t))}, \eta_{j}^{i}(\theta(t))=\prod_{j=1}^{l} \mu_{j}^{i}\left(\theta_{j}(t)\right)
$$

and $\mu_{j}^{i}\left(\gamma_{j}(t)\right)$ representing the

membership value of $\gamma_{j}(t)$ in $\mu_{j}^{i}$. Then, it is easy to see that for all $t$ the basic properties of T-S fuzzy rules $\eta_{i}(\gamma(t)) \geq 0, \sum_{i=1}^{r} \eta_{i}(\gamma(t))=1$.

The following lemmas are essential to get the main results and are stated below

Lemma 2.1 (Schur complement) given constant matrices $\Lambda_{1}, \Lambda_{2}, \Lambda_{3}$ with appropriate dimensions, where $\Lambda_{1}^{T}=\Lambda_{1}$ and $\Lambda_{2}{ }^{T}=\Lambda_{2}>0$, the inequality

$$
\Lambda_{1}+\Lambda_{3}^{T} \Lambda_{2}^{-1} \Lambda_{3}<0
$$

holds, if and only if 


$$
\left[\begin{array}{cc}
\Lambda_{1} & \Lambda_{3}^{T} \\
* & -\Lambda_{2}
\end{array}\right]<0, \text { or }\left[\begin{array}{cc}
-\Lambda_{2} & \Lambda_{3} \\
* & \Lambda_{1}
\end{array}\right]<0
$$

Lemma 2.2. [15] For any constant matrix $Q=Q^{T}>0$ and a scalar $r>0$ such that the following integrations are well defined, then

$$
\begin{gathered}
-\int_{t-r}^{r} x^{T}(\alpha) Q x(\alpha) d \alpha \leq-\frac{1}{r}\left(\int_{t-r}^{t} x(\alpha) d \alpha\right)^{T} Q\left(\int_{t-r}^{t} x(\alpha) d \alpha\right) \\
-\int_{-r}^{0} \int_{t+\theta}^{t} x^{T}(\alpha) Q x(\alpha) d \alpha d \theta \leq-\frac{2}{r^{2}}\left(-\int_{-r}^{0} \int_{t+\theta}^{t} x(\alpha) d \alpha d \theta\right)^{T} Q\left(-\int_{-r}^{0} \int_{t+\theta}^{t} x(\alpha) d \alpha d \theta\right) .
\end{gathered}
$$

Lemma 2.3.[18] Let $U, V(t), J$ and $K$ be real matrices of appropriate dimensions with $K$ satisfying $K=K^{T}$, then

$$
K+U V(t) J+J^{T} V^{T}(t) U^{T}<0, \quad V^{T}(t) V(t) \leq I
$$

if and only if there exist a scalar $\varepsilon>0$ such that

$$
K+\varepsilon^{-1} U U^{T}+\varepsilon J^{T} J<0 .
$$

We first investigate the stability analysis of T-S fuzzy system without parameter uncertainties (i.e., $\Delta A_{1 i}(t)=\Delta A_{2 i}(t)=\Delta A_{3 i}(t)=0$ ). The system can be reduced the form as

$$
\dot{x}(t)=\sum_{i=1}^{r} \mu_{i}(\gamma(t))\left\{A_{1 i} x(t)+A_{2 i} x(t-\tau(t))+A_{3 i} \int_{t-r(t)}^{t} x(\alpha) d \alpha\right\}
$$

By constructing an appropriate LKF, the delay-dependent stability condition is summarized in the following theorem.

Theorem 3.1. For given scalars $\tau_{1} \geq 0, \tau_{2}>0, r>0$, and $\mu$ the T-S fuzzy system described by (4) is asymptotically stable, if there exist positive-definite symmetric matrices $P>0, Q_{i}>0(i=1, \ldots, 5), R_{i}>0(i=1, \ldots, 5)$ and appropriate dimension matrices $M_{1}, M_{2}$ such that the following LMI holds

$$
\mho=\left[\begin{array}{llllllll}
\mho_{1,1} & \mho_{1,2} & \mho_{1,3} & \mho_{1,4} & \mho_{1,5} & \mho_{1,6} & \mho_{1,7} & \mho_{1,8} \\
* & \mho_{2,2} & \mho_{2,3} & \mho_{2,4} & \mho_{2,5} & \mho_{2,6} & \mho_{2,7} & \mho_{2,8} \\
* & * & \mho_{3,3} & \mho_{3,4} & \mho_{3,5} & \mho_{3,6} & \mho_{3,7} & \mho_{3,8} \\
* & * & * & \mho_{4,4} & \mho_{4,5} & \mho_{4,6} & \mho_{4,7} & \mho_{4,8} \\
* & * & * & * & \mho_{5,5} & \mho_{5,6} & \mho_{5,7} & \mho_{5,8} \\
* & * & * & * & * & \mho_{6,6} & \mho_{6,7} & \mho_{6,8} \\
* & * & * & * & * & * & \mho_{7,7} & \mho_{7,8} \\
* & * & * & * & * & * & * & \mho_{8,8}
\end{array}\right]<0
$$

where

$$
\mho_{1,1}=Q_{1}+Q_{2}+Q_{3}+\tau_{1}^{2} R_{1}+\tau_{2}^{2} R_{2}+r^{2} R_{3}-2 R_{4}-2 R_{5}+2 M_{1} A_{1 i},
$$




$$
\mho_{1,4}=M_{1} A_{2 i}, \quad \mho_{1,5}=P-M_{1}+A_{1 i}^{T} M_{2}^{T}, \quad \mho_{1,6}=M_{1} A_{3 i}, \quad \mho_{1,7}=\frac{2}{\tau_{1}} R_{4}, \quad \mho_{1,8}=
$$
$\frac{2}{\tau_{2}} R_{5}$

$\mu) Q_{4}$

$$
\mho_{2,2}=-Q_{2}+Q_{5}, \quad \mho_{3,3}=-Q_{1}-Q_{4}, \quad \mho_{4,4}=-(1-\mu) Q_{3}-(1-\mu) Q_{5}+(1-
$$

$$
\begin{aligned}
& \mho_{4,5}=A_{2 i}^{T} M_{2}^{T}, \quad \mho_{5,5}=\frac{1}{2} \tau_{1}^{2} R_{4}+\frac{1}{2} \tau_{2}^{2} R_{5}-2 M_{2}, \quad \mho_{5,6}=M_{2} A_{2 i}, \quad \mho_{6,6}=-R_{3}, \\
& \mho_{7,7}=-R_{1}-\frac{2}{\tau_{1}^{2}} R_{4}, \quad \mho_{8,8}=-R_{2}-\frac{2}{\tau_{2}^{2}} R_{5}
\end{aligned}
$$

and the terms which have not been stated here are zero.

\section{Proof:}

To derive the asymptotic stability conditions for delayed T-S fuzzy system(4), we consider the following Lyapunov-Krasovskii functional (LKF) as:

$$
V\left(x_{t}\right)=\sum_{i=1}^{4} V_{i}\left(x_{t}\right)
$$

where

$$
\begin{aligned}
& V_{1}\left(x_{t}\right)=x^{T}(t) P x(t), \\
& V_{2}\left(x_{t}\right)=\int_{t-\tau_{2}}^{t} x^{T}(\alpha) Q_{1} x(\alpha) d \alpha+\int_{t-\tau_{1}}^{t} x^{T}(\alpha) Q_{2} x(\alpha) d \alpha+\int_{t-\tau(t)}^{t} x^{T}(\alpha) Q_{3} x(\alpha) d \alpha \\
& \quad+\int_{t-\tau_{2}}^{t-\tau(t)} x^{T}(\alpha) Q_{4} x(\alpha) d \alpha+\int_{t-\tau(t)}^{t-\tau_{1}} x^{T}(\alpha) Q_{5} x(\alpha) d \alpha, \\
& V_{3}\left(x_{t}\right)=\tau_{1} \int_{-\tau_{1}}^{0} \int_{t+\theta}^{t} x^{T}(\alpha) R_{1} x(\alpha) d \alpha d \theta+\tau_{2} \int_{-\tau_{2}}^{0} \int_{t+\theta}^{t} x^{T}(\alpha) R_{2} x(\alpha) d \alpha d \theta \\
& \quad+r \int_{-r}^{0} \int_{t+\theta}^{t} x^{T}(\alpha) R_{3} x(\alpha) d \alpha d \theta \\
& V_{4}\left(x_{t}\right)=\int_{-\tau_{1}}^{0} \int_{\theta}^{0} \int_{t+\beta}^{t} \dot{x}^{T}(\alpha) R_{4} \dot{x}(\alpha) d \alpha d \beta d \theta+\int_{-\tau_{2}}^{0} \int_{\theta}^{0} \int_{t+\beta}^{t} \dot{x}^{T}(\alpha) R_{5} \dot{x}(\alpha) d \alpha d \beta d \theta
\end{aligned}
$$

Then, to obtain the asymptotic stability taking the time derivative of the $\operatorname{LKF} V\left(x_{t}\right)$ along the trajectory of the system (4). $\dot{V}\left(x_{t}\right)$ can be evaluated by using the above mentioned terms

$$
\begin{aligned}
\dot{V}_{1}\left(x_{t}\right) & =2 x^{T}(t) P \dot{x}(t) \\
\dot{V}_{2}\left(x_{t}\right) & \leq x^{T}(t)\left(Q_{1}+Q_{2}+Q_{3}\right) x(t)-x^{T}\left(t-\tau_{2}\right)\left(Q_{1}+Q_{4}\right) x\left(t-\tau_{2}\right) \\
& +x^{T}\left(t-\tau_{1}\right)\left(Q_{5}-Q_{2}\right) x\left(t-\tau_{1}\right)-(1-\mu) x^{T}(t-\tau(t))\left(Q_{3}-Q_{4}+Q_{5}\right) x(t-
\end{aligned}
$$

$\tau(t)),(7)$

$$
\begin{gathered}
\dot{V}_{3}\left(x_{t}\right) \leq x^{T}(t)\left(\tau_{1}^{2} R_{1}+\tau_{2}^{2} R_{2}+r^{2} R_{3}\right) x(t)-\tau_{1} \int_{t-\tau_{1}}^{t} x^{T}(\alpha) R_{1} x(\alpha) d \alpha \\
-\tau_{2} \int_{t-\tau_{2}}^{t} x^{T}(\alpha) R_{2} x(\alpha) d \alpha-r \int_{t-r}^{t} x^{T}(\alpha) R_{3} x(\alpha) d \alpha
\end{gathered}
$$

$$
\dot{V}_{4}\left(x_{t}\right) \leq \frac{1}{2} \dot{x}^{T}(t)\left(R_{4}+R_{5}\right) \dot{x}(t)-\int_{-\tau_{1}}^{0} \int_{t+\theta}^{0} \dot{x}^{T}(\alpha) R_{4} \dot{x}(\alpha) d \alpha d \theta-\int_{-\tau_{2}}^{0} \int_{t+\theta}^{0} \dot{x}^{T}(\alpha) R_{5} \dot{x}(\alpha) d \alpha d \theta
$$

By Lemma(2.2), from equations (8) and (9) we obtain

$$
-\tau_{1} \int_{t-\tau_{1}}^{t} x^{T}(\alpha) R_{1} x(\alpha) d \alpha \leq-\left(\int_{t-\tau_{1}}^{t} x^{T}(\alpha) d \alpha\right) R_{1}\left(\int_{t-\tau_{1}}^{t} x(\alpha) d \alpha\right)
$$




$$
\begin{aligned}
& -\tau_{2} \int_{t-\tau_{2}}^{t} x^{T}(\alpha) R_{2} x(\alpha) d \alpha \leq-\left(\int_{t-\tau_{2}}^{t} x^{T}(\alpha) d \alpha\right) R_{2}\left(\int_{t-\tau_{2}}^{t} x(\alpha) d \alpha\right) \\
& -r \int_{t-r}^{t} x^{T}(\alpha) R_{3} x(\alpha) d \alpha \leq-\left(\int_{t-r}^{t} x^{T}(\alpha) d \alpha\right) R_{3}\left(\int_{t-r}^{t} x(\alpha) d \alpha\right) \\
& -\int_{-\tau_{1}}^{0} \int_{t+\theta}^{0} \dot{x}^{T}(\alpha) R_{4} \dot{x}(\alpha) d \alpha d \theta \leq-\frac{2}{\tau_{1}^{2}}\left(\int_{\tau_{1}}^{0} \int_{t+\theta}^{0} \dot{x}^{T}(\alpha) d \alpha d \theta\right) R_{4}\left(\int_{-\tau_{1}}^{0} \int_{t+\theta}^{0} \dot{x}(\alpha) d \alpha d \theta\right) \\
& \leq-\frac{2}{\tau_{1}^{2}}\left[\tau_{1} x(t)-\int_{t-\tau_{1}}^{t} x(\alpha) d \alpha\right]^{T} R_{4}\left[\tau_{1} x(t)-\int_{t-\tau_{1}}^{t} x(\alpha) d \alpha\right]
\end{aligned}
$$

$$
\begin{aligned}
-\int_{-\tau_{2}}^{0} \int_{t+\theta}^{0} \dot{x}^{T}(\alpha) R_{5} \dot{x}(\alpha) d \alpha d \theta & \leq-\frac{2}{\tau_{2}^{2}}\left(\int_{\tau_{2}}^{0} \int_{t+\theta}^{0} \dot{x}^{T}(\alpha) d \alpha d \theta\right) R_{5}\left(\int_{-\tau_{2}}^{0} \int_{t+\theta}^{0} \dot{x}(\alpha) d \alpha d \theta\right) \\
& \leq-\frac{2}{\tau_{2}^{2}}\left[\tau_{2} x(t)-\int_{t-\tau_{2}}^{t} x(\alpha) d \alpha\right]^{T} R_{5}\left[\tau_{2} x(t)-\int_{t-\tau_{2}}^{t} x(\alpha) d \alpha\right]
\end{aligned}
$$

Consider a matrices $M_{1}, M_{2}$ of appropriate dimensions, the following equation holds:

$$
0=2\left[x^{T}(t) M_{1}+\dot{x}^{T}(t) M_{2}\right]\left[-\dot{x}(t)+A_{1 i} x(t)+A_{2 i} x(t-\tau(t))+A_{3 i} \int_{t-r}^{t} x(\alpha) d \alpha\right]
$$

Add the right sides of equations from (6) to (15), $\dot{V}\left(x_{t}\right)$ can be expressed as follows:

$$
\dot{V}\left(x_{t}\right) \leq \xi^{T}(t) \Phi \xi(t)
$$

where

$$
\begin{aligned}
\xi^{T}(t)=\left[x^{T}(t)\right. & x^{T}\left(t-\tau_{1}\right)^{T}(t \\
& \left.\left.-\tau_{2}\right) x^{T}(t-\tau(t)) \dot{x}^{T}(t) \int_{t-r}^{t} x^{T}(\alpha) d \alpha \int_{t-\tau_{1}}^{t} x^{T}(\alpha) d \alpha \int_{t-\tau_{2}}^{t} x^{T}(\alpha) d \alpha\right] .
\end{aligned}
$$

From (5), we can conclude that $\mho<0$, therefore, the system (4) is asymptotically stable in the Lyapunov sense without uncertainties.

Remark 3.2 Consider the following T-S fuzzy with the exemption of distributed delay only having

constant delay in (4)

$$
\begin{aligned}
& \dot{x}(t)=\sum_{i=1}^{r} \mu_{i}(\gamma(t))\left\{A_{1 i} x(t)+A_{2 i} x(t-\tau)\right\} \\
& x(t)=\phi(t), \quad \forall t \in[-\tau, 0],
\end{aligned}
$$

For this system we have the following corollary.

Corollary 3.3 For given scalar $\tau>0$, the T-S fuzzy system described by (17) is asymptotically stable, if there exist positive-definite symmetric matrices $P>0, Q_{i}>0(i=1,2,3)$ and appropriate dimension matrix $M_{3}$ such that the following LMI holds

$$
\Omega=\left[\begin{array}{llll}
Q_{1}+\tau^{2} Q_{2}-2 \tau^{2} Q_{3} & 0 & P+A_{1 i}^{T} M_{3}^{T} & -2 \tau Q_{3} \\
* & -Q_{2} & A_{2 i}^{T} M_{3}^{T} & 0 \\
* & * & -2 M_{3}+\frac{1}{2} Q_{3} & 0 \\
* & * & * & -Q_{2}-2 Q_{3}
\end{array}\right]<0
$$


Proof. Consider the new LKF

$$
\begin{aligned}
V\left(x_{t}\right)= & x^{T}(t) P x(t)+\int_{t-\tau}^{t} x^{T}(s) Q_{1} x(\alpha) d \alpha+\tau \int_{-\tau}^{0} \int_{t+\theta}^{t} x^{T}(s) Q_{2} x(\alpha) d \alpha d \theta \\
& +\tau^{2} \int_{-\tau}^{0} \int_{\theta}^{0} \int_{t+\beta}^{t} \dot{x}^{T}(\alpha) Q_{3} \dot{x}(\alpha) d \alpha d \beta d \theta
\end{aligned}
$$

Taking the time derivative of the LKF along the trajectory of the system (17) and consider the zero equation $0=2 \dot{x}^{T}(t) M_{3}\left[-\dot{x}(t)+A_{1 i} x(t)+A_{2 i} x(t-\tau)\right]$, the proof of this corollary is similar to Theorem 3.1 and we can show that $\dot{V}\left(x_{t}\right) \leq \Xi_{1}^{T} \Omega \Xi_{1}<0$.

In the previous corollary, T-S fuzzy stability criteria with constant delay have been derived based on LMI for the system (4).

Next, we will obtain the robust asymptotic stability, in order to get robust stability we consider the uncertain T-S fuzzy system by using the results of Theorem 3.1 with uncertainty conditions.

In this section, based on parameter uncertainties (2), we derive the robust stability condition of the uncertain T-S fuzzy system based on Theorem 1.

Theorem 4.1 For given scalars $\tau_{1} \geq 0, \tau_{2}>0, \quad r>0, \quad 0<\mu<1$, the T-S fuzzy (3) is robustly asymptotically stable, if there exist positive definite matrices $P>0, Q_{i}>0(i=$ $1, \ldots, 5), R_{i}>0(i=1, \ldots, 5)$, for any real matrices $M_{1}, M_{2}$ of appropriate dimensions, and a positive scalar $\varepsilon_{i}$ such that the following LMI is feasible

$$
\bar{\mho}=\left[\begin{array}{lll}
\mho & M^{T} B_{i} & \varepsilon_{i} D_{i} \\
* & -\varepsilon_{i} I & 0 \\
* & * & -\varepsilon_{i} I
\end{array}\right]<0,
$$

with

$$
\begin{aligned}
M^{T} & =\left[\begin{array}{llllllll}
M_{1}^{T} & 0 & 0 & 0 & M_{2}^{T} & 0 & 0 & 0
\end{array}\right]^{T}, \\
D_{i}^{T} & =\left[\begin{array}{llllllll}
D_{1 i}^{T} & 0 & 0 & D_{2 i}^{T} & 0 & D_{3 i}^{T} & 0 & 0
\end{array}\right]^{T},
\end{aligned}
$$

and $\mho$ is defined as in Theorem 3.1.

Proof. Consider the LKF as same as in Theorem 3.1. Replace $A_{1 i}, A_{2 i}, A_{3 i}$ in the LMI (5) with $A_{1 i}+\Delta A_{1 i}(t), A_{2 i}+\Delta A_{2 i}(t), A_{3 i}+\Delta A_{3 i}(t)$ respectively. Using Lemma 2.3, the matrix inequality given in(19) is equivalent to the following inequality

$$
\mho+\varepsilon_{i}^{-1}\left(M^{T} B_{i}\right)\left(M^{T} B_{i}\right)^{T}+\varepsilon_{i} D_{i}^{T} D_{i}<0,
$$

the inequality(20) is true for $\varepsilon_{i}>0$. Then, the proof of Theorem 4.1 can be obtained by following the proof of Theorem 3.1 in a similar way.

\section{RESULTS}

In this section, we validate the derived delay-dependent conditions with following examples, which show the effectiveness of established theoretical results.

Example 5.1 Consider the T-S fuzzy time varying delay system (4) without parameter uncertainties and the following matrix parameters

$$
A_{11}=\left[\begin{array}{ll}
-3 & 0 \\
0.1 & -4
\end{array}\right], \quad A_{21}=\left[\begin{array}{ll}
-1 & 0 \\
-1 & -1
\end{array}\right], \quad A_{31}=\left[\begin{array}{ll}
-2 & 0 \\
0 & -3
\end{array}\right], \quad A_{12}=\left[\begin{array}{ll}
-6 & 0 \\
0 & -7.5
\end{array}\right],
$$




$$
A_{22}=\left[\begin{array}{ll}
-1.6 & 0 \\
0 & -5
\end{array}\right], \quad A_{32}=\left[\begin{array}{ll}
-2 & 0.1 \\
0 & -2.1
\end{array}\right] \text {. }
$$

Let $\tau_{1}=0.01, \tau_{2}=0.58, \mu=0.1$ and $r=0.1$ in Theorem 3.1, then the following feasible solutions can be obtained by the use of MATLAB toolbox

$$
\begin{gathered}
P=\left[\begin{array}{ll}
6.7059 & -0.2764 \\
-0.2764 & 2.8521
\end{array}\right], \quad Q_{1}=\left[\begin{array}{ll}
2.2969 & -0.2187 \\
-0.2187 & 1.3009
\end{array}\right], \quad Q_{2}=\left[\begin{array}{ll}
7.8833 & -0.4215 \\
-0.4215 & 7.2938
\end{array}\right], \\
Q_{3}=\left[\begin{array}{ll}
5.4570 & -0.4111 \\
-0.4111 & 5.8649
\end{array}\right], \quad Q_{4}=\left[\begin{array}{ll}
2.5534 & -0.2115 \\
-0.2115 & 1.3482
\end{array}\right], \quad Q_{5}=\left[\begin{array}{ll}
5.1065 & -0.1558 \\
-0.1558 & 5.8496
\end{array}\right], \\
R_{1}=\left[\begin{array}{ll}
6.8232 & -0.0013 \\
-0.0013 & 6.8169
\end{array}\right], \quad R_{2}=\left[\begin{array}{ll}
2.6476 & -0.2512 \\
-0.2512 & 2.2437
\end{array}\right], \quad R_{3}=\left[\begin{array}{ll}
13.6752 & 0.3428 \\
0.3428 & 19.2104
\end{array}\right], \\
R_{4}=1.0 e-04 *\left[\begin{array}{ll}
0.2980 & 0.0008 \\
0.0008 & 0.3020
\end{array}\right], \quad R_{5}=\left[\begin{array}{ll}
1.7796 & 0.0962 \\
0.0962 & 0.7568
\end{array}\right] .
\end{gathered}
$$

This shows that the system (4) is asymptotically stable in the Lyapunov sense.

Example 5.2 Consider the T-S fuzzy time varying delay system (3) with parameter uncertainties and the following matrices

$$
\begin{aligned}
A_{11} & =\left[\begin{array}{ll}
-3 & 0 \\
0.1 & -4
\end{array}\right], \quad A_{21}=\left[\begin{array}{ll}
-1 & 0 \\
-1 & -1
\end{array}\right], \quad A_{31}=\left[\begin{array}{ll}
-2 & 0 \\
0 & -3
\end{array}\right], \quad A_{12}=\left[\begin{array}{ll}
-6 & 0 \\
0 & -7.5
\end{array}\right], \\
A_{22} & =\left[\begin{array}{ll}
-1.6 & 0 \\
0 & -5
\end{array}\right], \quad A_{32}=\left[\begin{array}{ll}
-2 & 0.1 \\
0 & -2.1
\end{array}\right], \quad B_{1}=B_{2}=\left[\begin{array}{ll}
1 & 0 \\
0 & 1
\end{array}\right], \\
D_{11} & =D_{12}=\left[\begin{array}{ll}
-1.6 & 0 \\
0 & -0.05
\end{array}\right], \quad D_{21}=\left[\begin{array}{ll}
-0.9 & 0 \\
0 & -0.7
\end{array}\right], \quad D_{22}=\left[\begin{array}{ll}
-0.1 & 0 \\
0 & -0.4
\end{array}\right], \\
D_{31} & =\left[\begin{array}{ll}
-0.1 & 0 \\
0 & -0.5
\end{array}\right], \quad D_{32}=\left[\begin{array}{ll}
0.15 & 0.2 \\
0 & -0.04
\end{array}\right], \quad I=\left[\begin{array}{ll}
1 & 0 \\
0 & 1
\end{array}\right] .
\end{aligned}
$$

Let $\tau_{1}=0.1, \tau_{2}=0.2, \mu=0.1$ and $r=0.2$ in Theorem 4.1, then the following feasible solutions can be obtained by the use of MATLAB toolbox

$$
\begin{gathered}
P=\left[\begin{array}{ll}
113.8502 & -6.5989 \\
-6.5989 & 39.1794
\end{array}\right], \quad Q_{1}=\left[\begin{array}{ll}
16.6999 & -2.8940 \\
-2.8940 & 10.1159
\end{array}\right], \quad Q_{2}=\left[\begin{array}{ll}
94.7342 & -5.4236 \\
-5.4236 & 97.6428
\end{array}\right], \\
Q_{3}=\left[\begin{array}{ll}
60.1646 & -3.7025 \\
-3.7025 & 68.2741
\end{array}\right], \quad Q_{4}=\left[\begin{array}{ll}
19.6681 & -3.4870 \\
-3.4870 & 10.9062
\end{array}\right], \quad Q_{5}=\left[\begin{array}{ll}
75.5456 & -1.9613 \\
-1.9613 & 86.7265
\end{array}\right], \\
R_{1}=\left[\begin{array}{ll}
72.0249 & -0.7447 \\
-0.7447 & 70.9979
\end{array}\right], \quad R_{2}=\left[\begin{array}{ll}
65.9581 & -2.6348 \\
-2.6348 & 62.0863
\end{array}\right], \quad R_{3}=\left[\begin{array}{ll}
455.9001 & 16.6373 \\
16.6373 & 370.0059
\end{array}\right],
\end{gathered}
$$




$$
R_{4}=\left[\begin{array}{ll}
0.8232 & 0.0027 \\
0.0027 & 0.8237
\end{array}\right], \quad R_{5}=\left[\begin{array}{ll}
3.2559 & 0.0519 \\
0.0519 & 3.2355
\end{array}\right], \quad \varepsilon_{1}=91.8366, \quad \varepsilon_{2}=91.8366
$$

This shows that the system (3) is robustly asymptotically stable in the Lyapunov sense.

\section{CONCLUSION}

The stability problem of robust stability for the T-S fuzzy systems with mixed time-varying delays have been investigated. The mixed time-varying delays have considered for the proposed T-S fuzzy system based on the LKF which contains the information of time-varying delays(i.e. The upper and lower bounds ). The delay-dependent robust stability conditions investigated with LMIs. The obtained delay-dependent conditions ensure that the robustly asymptotically stability of the proposed delayed T-S fuzzy systems. Finally, numerical two examples are given to show the effectiveness of the derived sufficient conditions.

\section{REFERENCES}

[1] Yoneyama, J. (2007). Robust stability and stabilization for uncertain Takagiâ€"Sugeno fuzzy time-delay systems. Fuzzy sets and systems, 158(2), 115-134.

[2] Yoneyama, J. (2007). New delay-dependent approach to robust stability and stabilization for Takagiâ€"Sugeno fuzzy time-delay systems. Fuzzy Sets and Systems, 158(20), 2225-2237.

[3] Lien, C. H., Yu, K. W., Chen, W. D., Wan, Z. L., \& Chung, Y. J. (2007). Stability criteria for uncertain Takagi-Sugeno fuzzy systems with interval time-varying delay. IET Control Theory \& Applications, 1(3), 764-769.

[4] Lin, C., Wang, Q. G., \& Lee, T. H. (2006). Delay-dependent LMI conditions for stability and stabilization of T-S fuzzy systems with bounded time-delay. Fuzzy sets and systems, 157(9), 1229-1247.

[5] Manivannan, A., \& Muralisankar, S. (2016). Robust stability analysis of Takagiâ€"Sugeno fuzzy nonlinear singular systems with time-varying delays using delay decomposition approach. Circuits, Systems, and Signal Processing, 35(3), 791-809.

[6] Muralisankar, S., Manivannan, A., \& Gopalakrishnan, N. (2012). Asymptotic stability criteria for T-S fuzzy neural networks with discrete interval and distributed time-varying delays. Neural Computing and Applications, 21(1), 357-367.

[7] Peng, C., Tian, Y. C., \& Tian, E. (2008). Improved delay-dependent robust stabilization conditions of uncertain T-â€ $€$ "S fuzzy systems with time-varying delay. Fuzzy sets and systems, 159(20), 2713-2729.

[8] Wu, L., Su, X., Shi, P., \& Qiu, J. (2010). A new approach to stability analysis and stabilization of discrete-time T-S fuzzy time-varying delay systems. IEEE Transactions on Systems, Man, and Cybernetics, Part B (Cybernetics), 41(1), 273-286.

[9] Li, X., Yang, X., \& Song, S. (2019). Lyapunov conditions for finite-time stability of timevarying time-delay systems. Automatica, 103, 135-140.

[10] Liu, X., \& Zhang, K. (2019). Input-to-state stability of time-delay systems with delay-dependent impulses. IEEE Transactions on Automatic Control, 65(4), 1676-1682.

[11] Zhu, S., Chen, C., Yang, C., Fu, J., \& Zeng, Z. (2020). Finite-Time Stabilization and Energy Consumption Estimation for Delayed Nonlinear Systems. IEEE Transactions on Systems, Man, and Cybernetics: Systems.

[12] de Oliveira, F. S., \& Souza, F. O. (2020). Improved delay-dependent stability criteria for linear systems with multiple time-varying delays. International Journal of Control, 1-9. 
[13] Fei, W., Hu, L., Mao, X., \& Shen, M. (2017). Delay dependent stability of highly nonlinear hybrid stochastic systems. Automatica, 82, 165-170.

[14] Shao, H., Li, H., \& Shao, L. (2018). Improved delay-dependent stability result for neural networks with time-varying delays. ISA transactions, 80, 35-42.

[15] Sun, J., Liu, G. P., \& Chen, J. (2009). Delayâ€• dependent stability and stabilization of neutral timeâ€• delay systems. International Journal of Robust and Nonlinear Control: IFACâ€• Affiliated Journal, 19(12), 1364-1375.

[16] Aghayan, Z. S., Alfi, A., \& Machado, J. T. (2021). Robust stability analysis of uncertain fractional order neutral-type delay nonlinear systems with actuator saturation. Applied Mathematical Modelling, 90, 1035-1048.

[17] Juarez, L., Alexandrova, I. V., \& Mondie, S. (2020). Robust stability analysis for linear systems with distributed delays: A timeâ€• domain approach. International Journal of Robust and Nonlinear Control, 30(18), 8299-8312.

[18] Singh, V.: Robust stability of cellular neural networks with delay: linear matrix inequality approach, IEE Proceedings Control Theory and Applications 151, 125-129 (2004). 